\title{
Zebrafish as a Model to Study the Role of Peroxisome Proliferating-Activated Receptors in Adipogenesis and Obesity
}

\author{
Marjo J. Den Broeder, ${ }^{1}$ Victoria A. Kopylova, ${ }^{1}$ Leonie M. Kamminga, ${ }^{2,3}$ and Juliette Legler ${ }^{1}$ \\ ${ }^{1}$ Institute for Environmental Studies, Faculty of Earth and Life Sciences, VU University, 1081 HV Amsterdam, Netherlands \\ ${ }^{2}$ Department of Molecular Biology, Faculty of Sciences, Radboud Institute for Molecular Life Science, Radboud University, \\ Nijmegen, Netherlands \\ ${ }^{3}$ Radboud University Nijmegen Medical Center, Nijmegen, Netherlands
}

Correspondence should be addressed to Juliette Legler; juliette.legler@vu.nl

Received 17 August 2015; Revised 29 October 2015; Accepted 5 November 2015

Academic Editor: Stéphane Mandard

Copyright (C) 2015 Marjo J. Den Broeder et al. This is an open access article distributed under the Creative Commons Attribution License, which permits unrestricted use, distribution, and reproduction in any medium, provided the original work is properly cited.

\begin{abstract}
The Peroxisome Proliferator-Activated Receptors (PPARs) PPARA and PPARD are regulators of lipid metabolism with important roles in energy release through lipid breakdown, while PPARG plays a key role in lipid storage and adipogenesis. The aim of this review is to describe the role of PPARs in lipid metabolism, adipogenesis, and obesity and evaluate the zebrafish as an emerging vertebrate model to study the function of PPARs. Zebrafish are an appropriate model to study human diseases, including obesity and related metabolic diseases, as pathways important for adipogenesis and lipid metabolism which are conserved between mammals and fish. This review synthesizes knowledge on the role of PPARs in zebrafish and focuses on the putative function of PPARs in zebrafish adipogenesis. Using in silico analysis, we confirm the presence of five PPARs (pparaa, pparab, pparda, ppardb, and pparg) in the zebrafish genome with 67-74\% identity to human and mouse PPARs. During development, pparda/b paralogs and pparg show mRNA expression around the swim bladder and pancreas, the region where adipocytes first develop, whereas pparg is detectable in adipocytes at 15 days post fertilization (dpf). This review indicates that the zebrafish is a promising model to investigate the specific functions of PPARs in adipogenesis and obesity.
\end{abstract}

\section{Introduction}

In the last 30 years, obesity has become a worldwide epidemic, and according to the World Health Organization (WHO), over 700 million people can be characterized as obese [1]. Obesity is a risk factor for developing type II diabetes mellitus, cardiovascular diseases and hypertension, as well as cancer. Over the last decade, many studies have described the important biological functions of Peroxisome Proliferator-Activated Receptors (PPARs) and their role in obesity. Genetic variation in PPARs results in altered fat deposition and body weight, as studies on the development of obesity, T2DM, dyslipidemia, and cardiovascular diseases have identified single nucleotide polymorphisms (SNPs) in PPAR genes [2]. PPARs are important factors for adipocyte differentiation and energy homeostasis and are highly expressed in tissue with active lipid metabolism. In addition, PPARs are involved in embryonic development, cell differentiation, and inflammation [3-5].

The aim of this review is to describe the function of PPARs in lipid metabolism and adipogenesis, in particular their role in obesity and related disorders. As numerous animal models have been used to study the origin of obesity and to gain better knowledge of PPAR-related molecular mechanisms, we evaluate the zebrafish as an emerging model to study adipogenesis. We hypothesize that PPARs in zebrafish have similar functions as in mammals.

(1) Peroxisome Proliferator-Activated Receptors. PPARs are nuclear hormone receptors which belong to the NR1C subfamily of steroid receptor superfamily. The classifications of PPARs in the nuclear receptor family are described in a nomenclature system according to Nuclear Receptors Nomenclature Committee [6]. Three PPARs have been 
identified in vertebrates, namely, PPARA (hPPAR, PPAR $\alpha$, and NR1C1), PPARD (NR1C2, PPAR $\beta / \delta$, FAAR, NUCI, and NUCII), and PPARG (NR1C3, PPAR $\gamma$ ) which are encoded by different genes [4]. The zebrafish orthologs of PPARA, PPARD, and PPARG are referred to as Pparaa, Pparab, Pparda, Ppardb, and Pparg according to the ZFIN nomenclature (http://zfin.org/).

Like other nuclear receptors, PPARs have a protein structure that generally consists of four parts, namely, an $\mathrm{N}$ terminal domain (NTD), a DNA-binding domain (DBD), a ligand binding domain (LBD), and a connective structure (hinge) [7, 8]. The NTD contains a ligand-independent activation factor-1 (AF-1). The DBD consists of two zinc fingers that specifically bind to the peroxisome proliferator response element (PPRE) in the promotor regions of PPAR target genes [9]. The C-terminal part of the protein, the LBD, consists of $13 \alpha$-helices and $4 \beta$-sheets which form the ligand binding pocket. The LBD contains the ligand dependent activation factor-2 (AF-2), and ligand binding to AF-2 results in activation of the $\operatorname{LBD}[3,10]$. Expression of PPARs is predominantly in the nucleus, though PPARA and PPARG are also found in lower concentrations in the cytoplasm as well $[11,12]$. PPARs can be shuttled between the cytoplasm and the nucleus by export receptors that recognize two different nuclear localization signals (NLSs) on the PPAR protein [12].

PPARs regulate transcriptional gene activation through heterodimerization with retinoid $\mathrm{X}$ receptors ( $\mathrm{RXR})[13,14]$. All PPARs can form a complex with RXR, and heterodimerization between PPAR and RXR is ligand independent [15, 16]. In the absence of ligands, PPAR:RXR heterodimers bound to PPREs will act as a transcriptional repressor due to binding of corepressor proteins such as nuclear receptor corepressor 1 (NCoR) and silencing mediator of retinoic acid and thyroid hormone receptor (SMRT). Binding of a specific PPAR agonist to LBD leads to the release of corepressor complex and recruitment of the coactivation factors. Coactivators of PPARs include the steroid receptor coactivator-1 (SRC-1), CREB-binding protein (CBP), PPARbinding protein (PBP), P300, cyclin G2, PPAR-interacting protein (PRIP), and PPAR $\gamma$ coactivator-1 (PGC-1) [17-19]. As a consequence of a conformational change of the LBD, the PPAR:RXR heterodimer binds to the PPRE present in promoter regions in order to regulate transcription of target genes by facilitating RNA polymerase II function. The PPRE consists of direct repeats (DRs) containing two hexanucleotide sequences AGGTCA which are separated by one nucleotide [20]. The $5^{\prime}$ flanking site of the PPRE is important for PPAR isoform:RXR heterodimer binding specificity and consists of a 7-nucleotide sequence consensus (C[A/G][A/G]A[A/T]CT) [21].

The LBD of the PPARs is activated by specific endogenous agonists such as fatty acids, fatty acids derivatives, phospholipids, eicosanoids, and prostaglandins (Table 1). Many synthetic ligands of PPARS have been developed for the treatment of diabetes mellitus and high levels of triglycerides and cholesterol, such as the thiazolidine drugs (TZDs) and fibrates (Table 1). Recently, chemicals present in the environment through human activity such as pesticides and phthalate ester plasticizers have also been identified as PPAR ligands $[22,23]$.

(2) Biological Function of PPARs in Lipid Metabolism and Adipocyte Differentiation. PPARA is mainly involved in fatty acid oxidation and is highly expressed in tissue with mitochondrial and peroxisomal $\beta$-oxidation such as brown adipose tissue (BAT), liver, and to a lower extent also in heart, kidney, and muscles [24]. PPARA is activated by long chain unsaturated fatty acids, eicosanoids, and synthetic fibrates, which have been developed to treat dyslipidemia by reducing triglyceride levels $[25,26]$.

PPARD is ubiquitously expressed and has a common function in fatty acid metabolism comparable to PPARA. PPARD also seems to be involved in embryo implantation, keratinocyte differentiation, and wound healing [27, 28]. PPARD is a promising drug target for heart defects caused by diabetes. A recent study has shown that PPARD induces glucose transport to the heart muscle cells and that diabetes patients have decreased PPARD expression in cardiac muscle when glycemic levels are high [29]. Synthetic agonists for PPARD have been developed for the treatment of obesity and related conditions, as overexpression increases glucose influx, reduces damage, improves insulin sensitivity, and reduces lipid accumulation [30].

In humans and mice, the PPARG gene has three splice variants: PPARG1, PPARG2, and PPARG3. PPARG1 and PPARG3 translate into identical proteins whereas PPARG2 contains an extra 28 amino acid regions in the NTD due to alternative splicing [31]. PPARG2 is an essential regulator of adipocyte differentiation and lipid storage in WAT, while PPARG1/3 has a more general function in lipid metabolism and is expressed in the colon and macrophages [32,33]. Many synthetic agonists have also been designed for PPARG2, with the most well known being the TZDs and fibrates, both used for the treatment of metabolic disorders like type 2 diabetes mellitus (T2DM). TZDs that target PPARG are used to increase insulin sensitivity and reduce glycemia in the treatment of diabetes, and, additionally, PPARG activation has shown protective effects on the vasculature [34]. Activation of PPARG by TZDs has also been linked to increased weight gain in patients based on PPARG function in adipocyte differentiation and its involvement in lipid homeostasis [35]. As PPARG has also been shown to regulate certain processes in cancer development, it might be a possible additional target in the treatment of cancer. New generation drugs that target both PPARA and PPARG are very promising and have already been used in treatment, as they show hypolipidemic, hypotensive, anti-inflammatory, and antiatherogenic action [29, 36, 37].

\section{Zebrafish as Model for Obesity}

Numerous animal models have been used to study the etiology of obesity in order to gain better understanding of molecular mechanisms and possible treatments. In the last decade, zebrafish (Danio rerio), shown in adult and larval stages in Figure 1, have emerged as an excellent model to study human diseases [38] due to a number of advantages 
TABLE 1: Endogenous and synthetic ligands of vertebrate PPARs.

\begin{tabular}{|c|c|c|}
\hline PPAR & Ligand type & Potential agonists \\
\hline \multirow[t]{2}{*}{ PPARA } & Endogenous & $\begin{array}{l}\text { Fatty acids (lauric acid, linoleic acid, linolenic acid, arachidonic acid, eicosapentaenoic acid (Xenopus), } \\
\text { docosahexaenoic acid (Xenopus), petroselinic acid (Xenopus), oleic acid (Xenopus), and elaidic acid } \\
(\text { Xenopus) }[66,67] \\
\left.\text { Eicosanoids (fatty acid-derived) (e.g., leukotriene } \mathrm{B}_{4}\right)[68,69] \\
\text { Prostaglandin J2 (fatty acid-derived) }[70] \\
\text { Endocannabinoids [71] }\end{array}$ \\
\hline & Synthetic & $\begin{array}{l}\text { Nonsteroidal anti-inflammatory drugs (NSAIDs) [72] } \\
\text { Fibrates (e.g., gemfibrozil, Bezafibrate, clofibrate, fenofibrate, ciprofibrate, pirinixic acid (Wy 14643), and } \\
\text { GW2331) }[3,67,73,74] \\
\text { ETYA }(5,8,11,14 \text {-eicosatetraynoic acid) (Xenopus) }[66]\end{array}$ \\
\hline \multirow{2}{*}{ PPARD } & Endogenous & $\begin{array}{l}\text { Fatty acids (e.g., docosahexaenoic acid, linoleic acid) [69] } \\
\text { Very Low Density Lipoproteins (VLDL) components [75] }\end{array}$ \\
\hline & Synthetic & $\begin{array}{l}\text { Fibrates (Wy-14,643, Bezafibrate) [69] } \\
\text { GW501516, GW800644 [76, 77] }\end{array}$ \\
\hline \multirow[b]{2}{*}{ PPARG } & Endogenous & $\begin{array}{l}\text { Fatty acids (docosahexaenoic acid, linoleic acid (mouse)) }[14,69] \\
\text { Prostaglandin J2 metabolite 15-deoxy-delta 12,14-PGJ2 }[70,78]\end{array}$ \\
\hline & Synthetic & $\begin{array}{l}\text { NSAIDs [72] } \\
\text { Thiazolidinediones [79] } \\
\text { ETYA }(5,8,11,14 \text {-eicosatetraynoic acid) (mouse) }[14] \\
\text { Prostaglandin J2 derivatives }[70,78] \\
\text { Fibrates (Bezafibrate, clofibrate, and GW2331) }[14,67]\end{array}$ \\
\hline
\end{tabular}

of this system. Zebrafish develop rapidly and have a short life cycle and detailed genome sequence information is available. The availability of new genome editing techniques like TALENs [39] and CRISPR-Cas9 [40], as well as several transgenesis tools (MultiSiteGateway Tol2, BAC transgenesis, Gal4/UAS, and Q system), enables the study of the function of genes. Zebrafish can also be used for high-throughput forward genetic and chemical screens [41-43], facilitating the identification of molecules that regulate biological functions.

Zebrafish are a promising model for obesity research, as lipid metabolism pathways are conserved between mammals and fish [44-46]. Zebrafish have the key organs that are important for energy homeostasis and metabolism in mammals, as well as other key functions such as appetite regulation in the brain [47], insulin regulation [48], endocrine signaling through leptin [49], and lipid storage in white adipocytes [50, 51]. Like humans, zebrafish kept on a high caloric diet show increased plasma triglyceride levels and hepatic steatosis as well as comparable expression patterns of genes involved in lipid metabolism such as LEP, SREBP, PPARA, PPARG, and $\mathrm{NR} 3 \mathrm{H} 1$ [45]. It has also been shown that obesity in zebrafish coincides with an increased plasma fibrinogen concentration that is induced via IL-6 and IL-1Bl secreted from visceral white adipose tissue. Zebrafish, as well as mice, rats, and humans, also produce a higher amount of IL- 6 and IL-1B upon high caloric feeding, adding to the evidence that major metabolic pathways between fish and mammals are very similar [45]. It is important to note, however, that zebrafish may not be an appropriate model for studying thermogenesis in higher vertebrates since brown adipose tissue has not been identified in poikilothermic animals. Zebrafish appear to not make thermogenic brown adipocytes, relying instead on thyroid hormone-mediated processes to generate heat in their muscles [52]. Although uncoupling proteins (UCPs 15) have been identified in zebrafish that are expressed in brain, liver, and muscle tissue but not in adipose tissue [53], differences in thermogenesis between mammals and fish are important to consider.

One major advantage of the zebrafish as a model of obesity is its optical transparence which allows temporal monitoring of adipocyte formation and fatty acid uptake in vivo [54]; adipocytes can be visualized in developing larvae with various dyes, including the sudanophilic dye Oil Red O (ORO) or Sudan dyes (Sudan III, Sudan IV, and Sudan Black B) and fluorescent dyes like Nile Red or LipidGreen (Figure 1(c)) [50, 55]. ORO and Sudan dyes are lipophilic (fat-soluble) dyes that can be applied as a soluble colorant for neutral lipids and cholesteryl esters. Nile Red binds to both neutral lipids and phospholipids while LipidGreen only binds to neutral lipids $[55,56]$. Dyes such as Sudan Black $\mathrm{B}$ and $\mathrm{ORO}$ are fixative based dyes, and fixation techniques are time consuming and may also cause deformation of lipid droplets in tissue [57], while Nile Red and LipidGreen can be applied in vivo. The potential of using Nile Red staining in zebrafish larvae as a whole-organism test for screening pharmaceuticals and toxicological agents has recently been demonstrated [58].

\section{Adipogenesis and Lipid Metabolism in Zebrafish}

Adipose tissue develops from pluripotent mesenchymal stem cells (MSCs) and commitment to this cell lineage gives rise to preadipocytes (determination phase) and subsequent terminal differentiation adipocytes (differentiation phase) (Figure $1(d)$ ) $[59,60]$. In the transcriptional cascade leading 


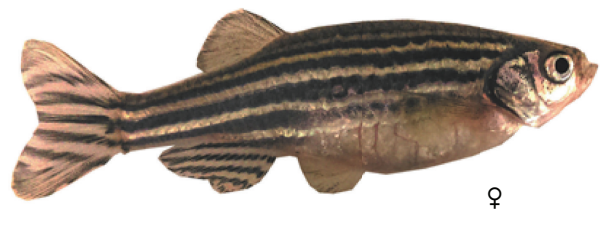

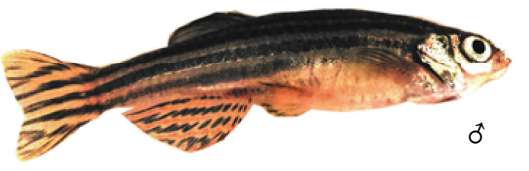

(a)

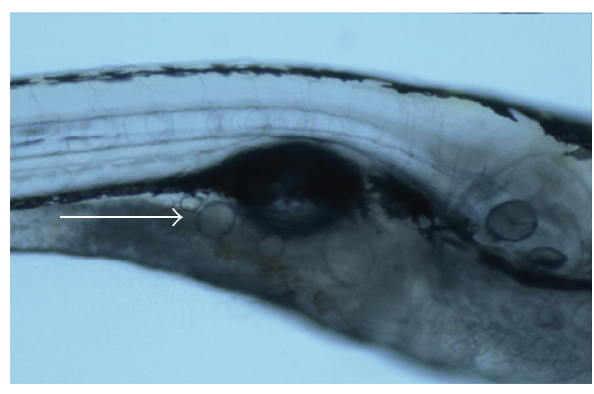

(c)

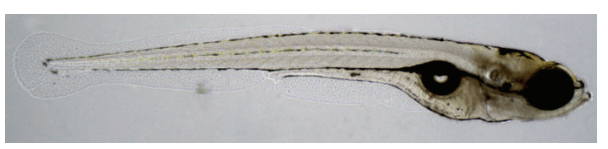

(b)

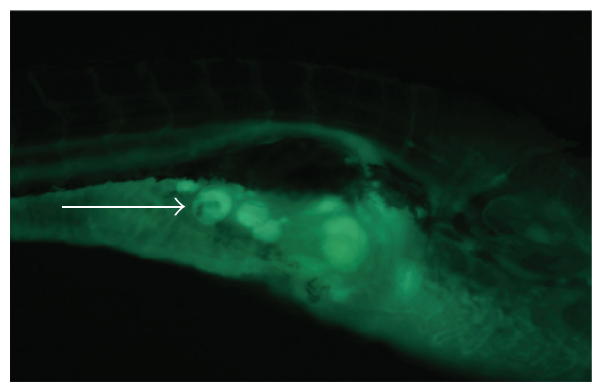

$\left(c^{\prime}\right)$

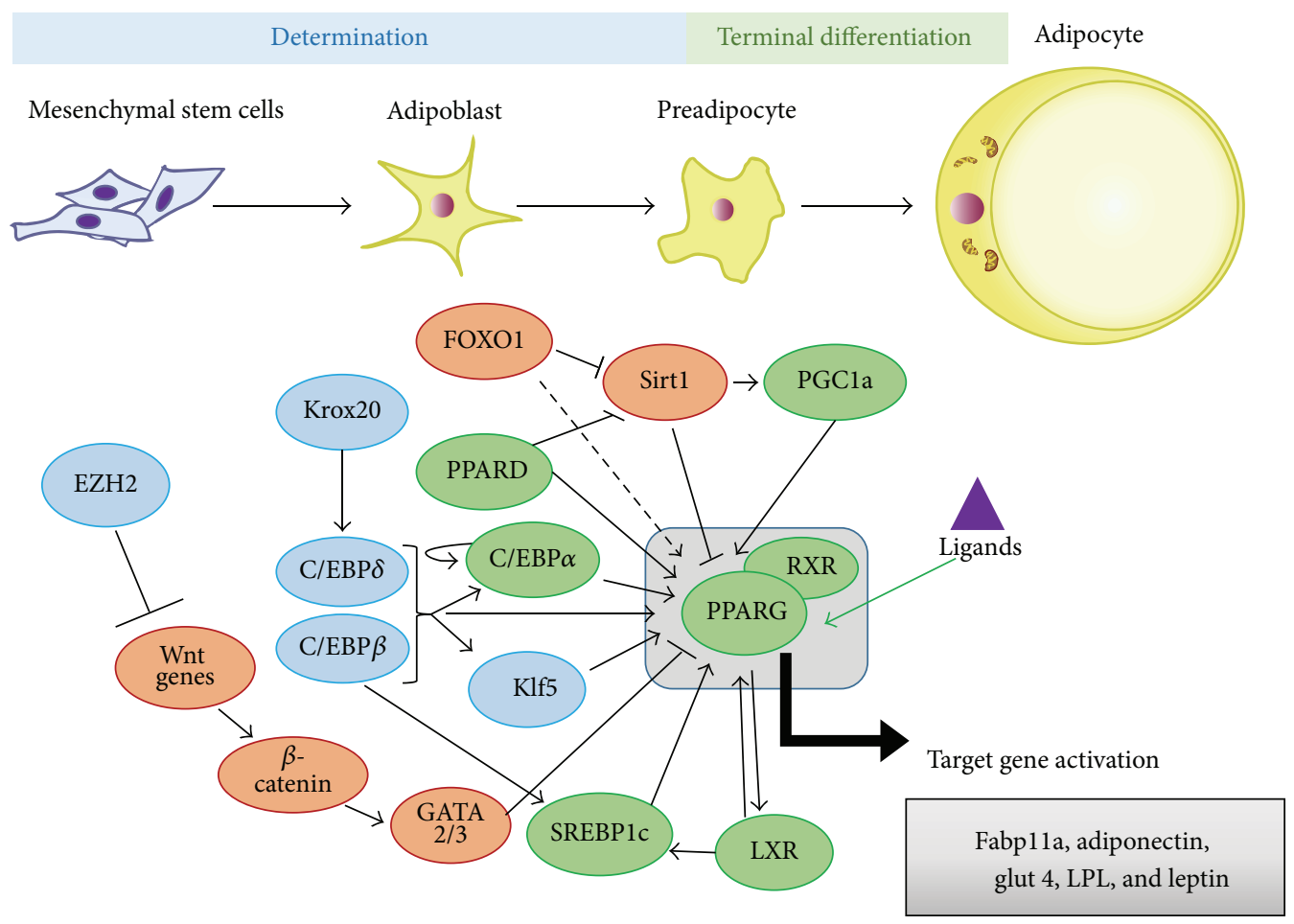

Early adipogenic factors

Adipogenic factors

Antiadipogenic factors

(d)

Figure 1: (a) An adult female and male zebrafish. (b) Developing zebrafish larvae (15 dpf). (c $\left.+c^{\prime}\right)$ A transmission light image of adipocytes in developing larvae (15 dpf) (left) and a fluorescent image after staining lipids with LipidGreen (right). (d) Transcriptional network of factors important for adipocyte differentiation in zebrafish and mammals. 
TABLE 2: Location and characteristics of the PPAR genes in zebrafish genome (GRCz10, Havana, and NCBI).

\begin{tabular}{|c|c|c|c|c|c|c|c|}
\hline PPAR & Gene & Chromosome location & GRCz10 & NCBI Nucleotide & cds (bp) & Protein (aa) & Exons \\
\hline pparaa & ENDARG00000031777 & 4 ; rev & CM002888.1 & NM_001161333 & 1,413 & 470 & 6 \\
\hline pparab & ENDARG00000054323 & 25 ; forw & СМ002909.1 & NM_001102567 & 1,380 & 459 & 7 \\
\hline pparda & ENDARG00000044525 & 22 ; forw & CM002906.1 & XM_694808, XM005168286 & 1491 & 496 & 7 \\
\hline ppardb & ENDARG00000009473 & 8 ; forw & CM002892.1 & NM_131468 & 1554 & 517 & 7 \\
\hline pparg & ENDARG00000031848 & 11 ; rev & CM002895.1 & NM_131467 & 1584 & 527 & 7 \\
\hline
\end{tabular}

to the differentiation of WAT (Figure $1(\mathrm{~d})$ ), the nuclear hormone receptor PPARG and CCAAT/enhancer-binding protein $(\mathrm{C} / \mathrm{EBP} \alpha)$ are key players. Two other members of the $\mathrm{C} / \mathrm{EBP}$ family, $\mathrm{C} / \mathrm{EBP} \beta$ and $\mathrm{C} / \mathrm{EBP} \delta$, are also important factors during differentiation into adipocytes [59, 61, 62]. $\mathrm{C} / \mathrm{EBP} \alpha$ is a transcription factor that regulates PPARG expression but also autoregulates its own expression. In zebrafish, orthologs of these genes are expressed in adipocytes and liver [51]. The terminal differentiation markers like leptin and fabplla are also expressed. Adiponectin has also been found in adult zebrafish adipose tissue [63].

During the first 4-5 days of development, zebrafish embryos are dependent on nutrients provided by the yolk sac which contains essential fat-soluble vitamins and triacylglycerol (TAG), as well as cholesterol. At days 5-6, the yolk sac is depleted and it is essential that larvae are provided with food to start to eat. The first signs of adipogenesis become visible at 8 days post fertilization (dpf) in the visceral cavity close to the pancreas but only in the minority of larvae. In most larvae, the first adipocytes translocate asymmetrically to the right visceral cavity [50]. Most adipocytes are observed from $12 \mathrm{dpf}$ onwards (standard length (SL) of $>5 \mathrm{~mm}$ ) in the pancreatic area [51]. In our laboratory, using LipidGreen staining, adipocytes are clearly visible at $15 \mathrm{dpf}$ in the visceral region in a subset of larvae (Figure $1(\mathrm{c})$ ). The amount of adipocytes is correlated with size of the larvae rather than age, suggesting body-length dependent lipid storage in adipocytes $[50,51]$. At $17 \mathrm{dpf}$, all larvae have WAT in the pancreatic and visceral area, indicating that visceral WAT development is not dependent on size but regulated by age. Subcutaneous (20 dpf, SL > 8,2 mm) and cranial (22 dpf, SL > 9,4 mm) adipocytes develop in a size-dependent manner [51]. Adult fish have the largest adipocyte deposits in the visceral regions, but smaller deposits are found subcutaneously in the tail and jaw, and in the periorbital regions. Zebrafish adipocytes are metabolically active and are able to communicate via gap junctions [64]. The adipocyte lipid droplet size can vary between 1 and $100 \mu \mathrm{m}$ and while most contain one large droplet, some may contain multiple smaller droplets in the early stage of development. These characteristics are conformant to mammalian adipocyte development and provide evidence that zebrafish contain WAT similar to mammals $[50,65]$.

\section{PPARs in Zebrafish}

The presence of five ppar genes in the zebrafish genome has been described previously [80-82]. We performed an in silico study to examine the homology of zebrafish PPARs with orthologs in different species. Using the TBLASTX search tool in Ensembl database (Ensembl GRCz10, http://www .ensembl.org/Danio_rerio/Info/Index), we checked for all five ppar genes in the newest version of zebrafish genome sequence available (GRC10). Based on the Havana/Ensembl merged sequences and the mammalian/zebrafish protein sequences, cDNA, and Nucleotide Database (NCBI), transcripts could be identified (Table 2). The zebrafish genome has undergone complete genome duplication in the teleost lineage after the divergence of fish and mammal ancestors $[83,84]$. Because of this, zebrafish have two PPARA (pparaa, pparab) and PPARD (pparda, ppardb) genes that are located on separate chromosomes (ohnologs). Only one PPARG (pparg) gene has been identified. pparaa is located on the reverse strand of chromosome 4 while pparab is located at forward strand of chromosome 25. The orthologs of PPARD are located on chromosome 22 (pparda) and chromosome 8 (ppardb) and are both positioned on the forward strand. In zebrafish, pparg gene is positioned on chromosome 11 on the reverse strand (Table 2). Comparing genomic regions of PPARs in human and zebrafish, we found conserved synteny regions using The Synteny Database [85]. pparaa and pparab showed both synteny to the same region at human $\mathrm{Chr}$ 22 and showed synteny between their location on zebrafish genome. Also, pparda and ppardb have synteny to the same location at human Chr 6, and as their PPARA orthologs. pparda and ppardb showed synteny between their location on zebrafish genome. The zebrafish genome surrounding pparg is highly conserved compared to human Chr 3 (Supplemental data $3 \mathrm{~A}-\mathrm{C}$ in Supplementary Material available online at http://dx.doi.org/10.1155/2015/358029). The conserved regions contain the same genes in both organisms, which indicates a conserved functional relationship between syntenic genes.

Pparaa and Pparab encode for proteins that are 470 and 459 amino acids (aa) long, respectively. Pparda consists of 496 aa, and Ppardb is 517 aa long. Pparg is the longest PPAR in zebrafish and is 527 aa in size. Protein alignment and the phylogenetic tree are based on 49 amino acid sequences from 15 different species (Supplemental Data 1). The protein sequences were subjected to homology analyses using PRALINE multiple sequence alignment (http://www.ibi.vu .nl/programs/pralinewww/) (Supplemental Data 1 and Supplemental Data 2). The phylogenetic tree was constructed using the software Geneious 9.0 (BLOSUM62 matrix, Genetic Distance model: Jukes-Cantor; Biomatters, http://www.geneious.com/) (Figure 2). All zebrafish PPARs show protein identity to human and mice orthologs, with similarity of human PPARA to zebrafish Pparab and Pparaa of $74 \%$ and 


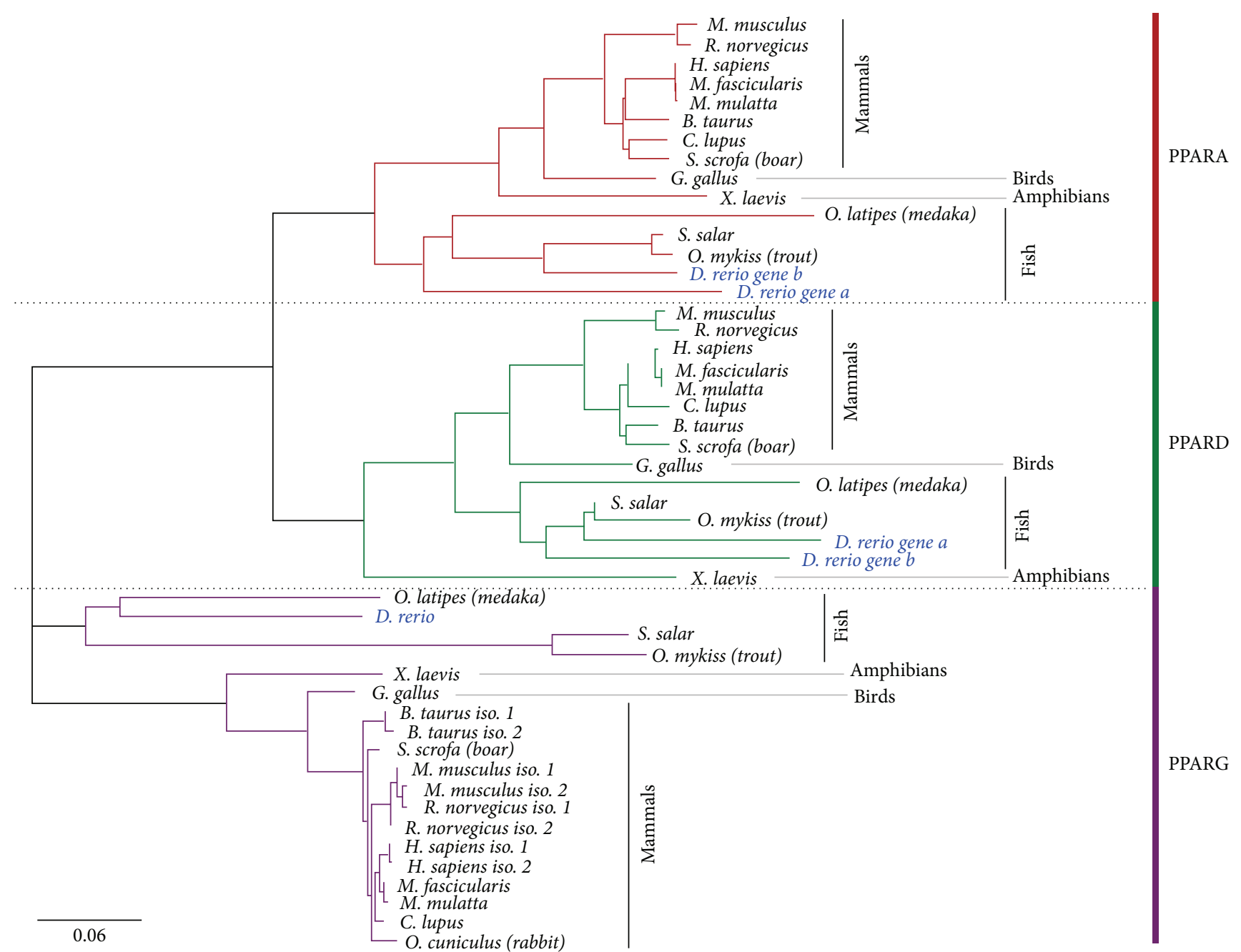

FIGURE 2: Phylogenetic tree of the PPAR orthologs in various organisms based on amino acid sequence difference (BLOSUM62 matrix). The phylogenetic tree is generated using the Geneious 9.0 software. Protein accession numbers information is provided in Supplemental Data 1.

67\%, respectively. Zebrafish Pparda and Ppardb showed 71\% and $73 \%$ similarity to human PPARD. Besides that, zebrafish Pparg is $67 \%$ similar to human PPARG (Table 3).

Amino acid sequence analysis of different homologues revealed that PPARs consist of a highly conserved protein family. The phylogenetic tree shows also that PPARA, PPARD, and PPARG proteins form distinct clusters of protein, although PPARA and PPARD were clustered together and the PPARG branch stands alone. The first gene duplication event of the PPAR gene family probably occurred in bony fish before being separated from birds and mammals. The second gene duplication is vertebrate specific and resulted in the different PPAR isotypes (A, D, and G) [86, 87]. After the gene duplication events, the PPARs acquired ligand binding capacities in an independent manner [88] and started to evolve by mutations in each PPARS resulting in refined specificity for ligands [87]. Zhao and colleagues [82] aligned the sequences of the DBD and LBD between human and 11 different species. The DBD and LBD for all PPAR are highly conserved between the different species, except for the DBD and LDB in Xenopus PPARD which are far much less conserved ( $83 \%$ and $75 \%$, resp.). One explanation can be that PPARD is less evolved in Xenopus compared to the other species and therefore has a separated position in the phylogenetic tree.

The zebrafish PPAR proteins contain several large regions of amino acids that are highly conserved (Supplemental Data 2). The N-terminal region of zebrafish PPARs is less similar, as this region is highly variable between organisms. The N-terminal region contains the conserved AF-1 that can be activated by endogenous factors like hormones and cytokines. In contrast, the AF-2 site located in the C-terminal region remains well conserved. Like human PPARs, zebrafish Pparaa, Pparab, and Pparg contain a threonine/serine-rich NLS2 site that is involved in cytoplasm-nucleus shuttling of the PPARs. This site was not found in Ppard ohnologs, however, which have a threonine/serine-rich part earlier in the protein which could possibly result in a similar action. The overall conservation of PPARG is fairly high between organisms, except for three fish species including the Japanese 
TABLE 3: Comparison of PPARs between human, mouse, and zebrafish.

\begin{tabular}{|c|c|c|c|}
\hline PPAR & Human & Mouse & Zebrafish \\
\hline \multicolumn{4}{|l|}{ PPARA } \\
\hline Genes & 1 & 1 & 2 \\
\hline Splice variants & 1 & 1 & 1 per gene \\
\hline Isoforms & 1 & 1 & 1 per gene \\
\hline Size (cds, bp) & 1,407 & 1,407 & $\begin{array}{l}\text { pparaa: } 1,413 \\
\text { pparab: } 1,380\end{array}$ \\
\hline Protein similarity to zebrafish & $\begin{array}{l}67 \% \text { for pparaa } \\
74 \% \text { for pparab }\end{array}$ & $\begin{array}{l}65 \% \text { for pparaa } \\
71 \% \text { for } p \text { parab }\end{array}$ & \\
\hline \multicolumn{4}{|l|}{ PPARD } \\
\hline Genes & 1 & 1 & 2 \\
\hline Splice variants & 1 & 1 & 1 per gene \\
\hline Isoforms & 1 & 1 & 1 per gene \\
\hline Size (cds, bp) & 1,326 & 1,323 & $\begin{array}{l}\text { pparda: } 1,491 \\
\text { ppardb: } 1,554\end{array}$ \\
\hline Protein similarity to zebrafish & $\begin{array}{l}71 \% \text { for } p p a r d a \\
73 \% \text { for } p \text { pardb }\end{array}$ & $\begin{array}{l}70 \% \text { for } p p a r d a \\
73 \% \text { for } p \text { pardb }\end{array}$ & \\
\hline \multicolumn{4}{|l|}{ PPARG } \\
\hline Genes & 1 & 1 & 1 \\
\hline Splice variants & 4 & 1 & 1 \\
\hline Isoforms & 2 & 2 & 1 \\
\hline Size (cds, bp) & $\begin{array}{l}\text { PPARG1 = 1,434 } \\
\text { PPARG2 = 1,518 }\end{array}$ & $\begin{array}{l}\text { PPARG1 }=1,428 \\
\text { PPARG2 }=1,518\end{array}$ & pparg: 1,584 \\
\hline Protein similarity to zebrafish & $67 \%$ & $67 \%$ & \\
\hline
\end{tabular}

medaka (Oryzias latipes), salmon (Salmo salar), and rainbow trout (Oncorhynchus mykiss), which have several (up to 5) additional amino acid regions throughout the protein. In all fish species, the LDB of PPARG is less conserved to human (74-78\%) and this can be explained by the rapid evolutionary rate of PPARG in teleosts [82]. When comparing human and zebrafish PPARG, two major differences between zebrafish Pparg and human PPARG were found. Firstly, the first 30 amino acids from the N-terminus differ highly between zebrafish Pparg and both hPPARG isoforms. In this region of the protein, hPPARG2 contains extra 28 amino acids compared to the hPPARG1 isoform. Conversely, zebrafish Pparg contains only 16 amino acids in this region that differ in all except two amino acids from hPPARG2. Secondly, zebrafish Pparg has an extra 27 amino acid regions starting from the 156th amino acid. Other fish species also have extra amino acid sequence so it is possible that, during evolution, teleost fish gained additional coding sequences which can have implications for protein folding and functionality. We also observed that all fish species analysed have an extra protein sequence in the ligand binding domain visible at amino acid position 401 in the protein alignment. This extra sequence is not a part of the ligand binding pocket and presumably should not interfere with binding to specific ligands. However, a previous study has shown differences in ligand binding specificity, as sequence comparison of zebrafish PPARg to human and mice PPARG revealed residual differences within the LDB [89].
The phylogenetic tree shows that the zebrafish PPARA ohnologs have evolved further from each other compared to the zebrafish PPARD ohnologs (Figure 2). The zebrafish PPARD ohnologs have most likely retained the same function (subfunctionalization), while the zebrafish PPARA ohnologs might have been subject to neofunctionalization [80]. pparab has more similarities with salmon and rainbow trout, while pparaa is on a separate branch in the phylogenetic tree and has a higher homology with Japanese medaka. Additionally, zebrafish PPARA and PPARD ohnologs seem to be more related to each other than to zebrafish PPARG.

In fish, PPARs have been identified and characterized but not much is known about their expression during developmental stages and adipogenesis. Zebrafish PPARs are expressed during development and so far little is known of the expression profiles of the paralogs pparaa, pparab, pparda, and ppardb. The limited expression studies that have been performed show that ppardb and pparg mRNA are already expressed early in development (5-10 hours post fertilization (hpf)) in whole embryo. From $20 \mathrm{hpf}$ on, the expression is more intensive in the head region and continues to be specifically expressed in the head, branchial arches, and pectoral fin at $36 \mathrm{hpf}$. From this time point, expression data for pparda is also available and comparable to that of ppardb and pparg. At $5 \mathrm{dpf}$, gene expression is detected around the swim bladder (pparda, ppardb, and pparg), in the liver (pparda, pparg), in the intestinal bulb (pparda), and also in the complete intestinal tract (pparg) [80]. The expression 
patterns of ppard paralogs and pparg can be an indication that they play a role in adipogenesis since it is known that first adipocytes arise in the pancreatic region underneath the swim bladder $[50,51]$. During the larval stage (15 dpf), pparg mRNA is localized in developing adipocytes within the pancreas and the intestinal epithelium in the same cells where Nile Red staining was observed [50]. Expression of the pparg gene is specifically detected in visceral adipose tissue and pancreas as opposed to liver [51].

\section{Conclusions}

PPARs are important factors in energy homeostasis and obesity development. While PPARA and PPARD are necessary for lipid breakdown, PPARG plays a role in lipid accumulation and adipogenesis. Single nucleotide polymorphisms in PPARs have been implicated in the development of obesity, T2DM, lipodystrophy, dyslipidemias, and cardiovascular risk. The zebrafish is an emerging model for research in obesity and related metabolic diseases and could be very useful for providing insight into the role of PPARs in the origins of obesity. All five PPAR genes (pparaa, pparab, pparda, ppardb, and pparg) show protein similarity to human and mice PPARs varying from 67 to $74 \%$. Three of the five zebrafish PPARs (pparda, ppardb, and pparg) show expression of mRNA in regions around swim bladder and pancreas that correlate to the sites where first adipocytes will develop, and at $15 \mathrm{dpf}$, pparg expression is detected in developing adipocytes. This colocalization suggests a role for PPARs in adipogenesis. However, detailed expression studies of PPARs during early stages of adipocyte development are not available, and to determine the subcellular expression of the different PPARs in zebrafish, specific antibody staining needs to be performed or transgenic fish lines should be generated. In addition, more conclusive evidence of the function of PPAR in zebrafish adipogenesis can be obtained by the generation of mutant lines. Taken together, this review shows that the zebrafish is a promising model for elucidating the specific functions of PPARs in adipogenesis and obesity.

\section{Conflict of Interests}

The authors declare that they do not have any conflict of interests.

\section{Acknowledgment}

This research is financially supported by Netherlands Organisation for Scientific Research (NWO) VIDI/864.09.005 and ASPASIA/015.006.018.

\section{References}

[1] WHO, "World health statistics 2012," World Health Organization, vol. 27, no. 1, pp. 179-187, 1949.

[2] E. L. Yong, J. Li, and M. H. Liu, "Single gene contributions: genetic variants of peroxisome proliferator-activated receptor (isoforms $\alpha, \beta / \Delta$ and $\gamma$ ) and mechanisms of dyslipidemias," Current Opinion in Lipidology, vol. 19, no. 2, pp. 106-112, 2008.
[3] J. Berger and D. E. Moller, "The mechanisms of action of PPARs," Annual Review of Medicine, vol. 53, no. 1, pp. 409-435, 2002.

[4] B. Desvergne and W. Wahli, "Peroxisome proliferator-activated receptors: nuclear control of metabolism," Endocrine Reviews, vol. 20, no. 5, pp. 649-688, 1999.

[5] K. Tachibana, D. Yamasaki, K. Ishimoto, and T. Doi, "The role of PPARs in cancer," PPAR Research, vol. 2008, Article ID 102737, 15 pages, 2008.

[6] Nuclear Receptors Nomenclature C, "A unified nomenclature system for the nuclear receptor superfamily," Cell, vol. 97, no. 2, pp. 161-163, 1999.

[7] M. M. Aagaard, R. Siersbæk, and S. Mandrup, "Molecular basis for gene-specific transactivation by nuclear receptors," Biochimica et Biophysica Acta-Molecular Basis of Disease, vol. 1812, no. 8, pp. 824-835, 2011.

[8] D. J. Mangelsdorf, C. Thummel, M. Beato et al., "The nuclear receptor superfamily: the second decade," Cell, vol. 83, no. 6, pp. 835-839, 1995.

[9] J. D. Tugwood, I. Issemann, R. G. Anderson, K. R. Bundell, W. L. McPheat, and S. Green, "The mouse peroxisome proliferator activated receptor recognizes a response element in the $5^{\prime}$ flanking sequence of the rat acyl CoA oxidase gene," The EMBO Journal, vol. 11, no. 2, pp. 433-439, 1992.

[10] V. Zoete, A. Grosdidier, and O. Michielin, "Peroxisome proliferator-activated receptor structures: ligand specificity, molecular switch and interactions with regulators," Biochimica et Biophysica Acta, vol. 1771, no. 8, pp. 915-925, 2007.

[11] T. Helledie, M. Antonius, R. V. Sørensen et al., "Lipid-binding proteins modulate ligand-dependent trans-activation by peroxisome proliferator-activated receptors and localize to the nucleus as well as the cytoplasm," Journal of Lipid Research, vol. 41, no. 11, pp. 1740-1751, 2000.

[12] T. Umemoto and Y. Fujiki, "Ligand-dependent nucleocytoplasmic shuttling of peroxisome proliferator-activated receptors, PPAR $\alpha$ and PPAR $\gamma$," Genes to Cells, vol. 17, no. 7, pp. 576-596, 2012.

[13] P. Tontonoz, R. A. Graves, A. I. Budavari et al., "Adipocytespecific transcription factor ARF6 is a heterodimeric complex of two nuclear hormone receptors, PPAR gamma and RXR alpha," Nucleic Acids Research, vol. 22, no. 25, pp. 5628-5634, 1994.

[14] P. Tontonoz, E. Hu, and B. M. Spiegelman, "Stimulation of adipogenesis in fibroblasts by PPAR $\gamma 2$, a lipid-activated transcription factor," Cell, vol. 79, no. 7, pp. 1147-1156, 1994.

[15] V. Chandra, P. Huang, Y. Hamuro et al., "Structure of the intact PPAR- $\gamma$-RXR- $\alpha$ nuclear receptor complex on DNA," Nature, vol. 456, no. 7220, pp. 350-356, 2008.

[16] N. Rochel, F. Ciesielski, J. Godet et al., "Common architecture of nuclear receptor heterodimers on DNA direct repeat elements with different spacings," Nature Structural and Molecular Biology, vol. 18, no. 5, pp. 564-570, 2011.

[17] V. Aguilar, J.-S. Annicotte, X. Escote, J. Vendrell, D. Langin, and L. Fajas, "Cyclin G2 regulates adipogenesis through PPAR gamma coactivation," Endocrinology, vol. 151, no. 11, pp. 52475254,2010

[18] Y. Zhu, C. Qi, C. Calandra, M. S. Rao, and J. K. Reddy, “Cloning and identification of mouse steroid receptor coactivator-1 (mSRC-1), as a coactivator of peroxisome proliferator-activated receptor gamma," Gene Expression, vol. 6, no. 3, pp. 185-195, 1996. 
[19] Y. Zhu, C. Qi, S. Jain, M. S. Rao, and J. K. Reddy, "Isolation and characterization of PBP, a protein that interacts with peroxisome proliferator-activated receptor," The Journal of Biological Chemistry, vol. 272, no. 41, pp. 25500-25506, 1997.

[20] S. A. Kliewer, K. Umesono, D. J. Mangelsdorf, and R. M. Evans, "Retinoid X receptor interacts with nuclear receptors in retinoic acid, thyroid hormone and vitamin D3 signalling," Nature, vol. 355, no. 6359, pp. 446-449, 1992.

[21] G. Venkatachalam, M. K. Sakharkar, A. P. Kumar, and M. V. Clement, "PPRESearch: peroxisome proliferator activator element search database," International Journal of Integrative Biology, vol. 8, no. 1, pp. 37-42, 2009.

[22] A. Janesick and B. Blumberg, "Minireview: PPAR $\gamma$ as the target of obesogens," Journal of Steroid Biochemistry and Molecular Biology, vol. 127, no. 1-2, pp. 4-8, 2011.

[23] A. Janesick and B. Blumberg, "Obesogens, stem cells and the developmental programming of obesity," International Journal of Andrology, vol. 35, no. 3, pp. 437-448, 2012.

[24] P. Escher, O. Braissant, S. Basu-Modak, L. Michalik, W. Wahli, and B. Desvergne, "Rat PPARs: quantitative analysis in adult rat tissues and regulation in fasting and refeeding," Endocrinology, vol. 142, no. 10, pp. 4195-4202, 2001.

[25] B. M. Forman, J. Chen, and R. M. Evans, "Hypolipidemic drugs, polyunsaturated fatty acids, and eicosanoids are ligands for peroxisome proliferator-activated receptors alpha and delta," Proceedings of the National Academy of Sciences of the United States of America, vol. 94, no. 9, pp. 4312-4317, 1997.

[26] K. Schoonjans, B. Staels, and J. Auwerx, "Role of the peroxisome proliferator-activated receptor (PPAR) in mediating the effects of fibrates and fatty acids on gene expression," Journal of Lipid Research, vol. 37, no. 5, pp. 907-925, 1996.

[27] Y. Barak, D. Liao, W. He et al., "Effects of peroxisome proliferator-activated receptor $\delta$ on placentation, adiposity, and colorectal cancer," Proceedings of the National Academy of Sciences of the United States of America, vol. 99, no. 1, pp. 303308, 2002.

[28] N. S. Tan, L. Michalik, N. Noy et al., "Critical roles of PPAR $\beta / \delta$ in keratinocyte response to inflammation," Genes and Development, vol. 15, no. 24, pp. 3263-3277, 2001.

[29] E. M. Burkart, N. Sambandam, X. Han et al., "Nuclear receptors $\operatorname{PPAR} \beta / \delta$ and PPAR $\alpha$ direct distinct metabolic regulatory programs in the mouse heart," The Journal of Clinical Investigation, vol. 117, no. 12, pp. 3930-3939, 2007.

[30] Y.-X. Wang, C.-H. Lee, S. Tiep et al., "Peroxisome-proliferatoractivated receptor $\gamma$ activates fat metabolism to prevent obesity," Cell, vol. 113, no. 2, pp. 159-170, 2003.

[31] L. Fajas, D. Auboeuf, E. Raspé et al., "The organization, promoter analysis, and expression of the human PPAR $\gamma$ gene," The Journal of Biological Chemistry, vol. 272, no. 30, pp. 1877918789, 1997.

[32] D. Ren, T. N. Collingwood, E. J. Rebar, A. P. Wolffe, and H. S. Camp, "PPAR $\gamma$ knockdown by engineered transcription factors: exogenous PPAR $\gamma 2$ but not PPAR $\gamma 1$ reactivates adipogenesis," Genes and Development, vol. 16, no. 1, pp. 27-32, 2002.

[33] G. Medina-Gomez, S. L. Gray, L. Yetukuri et al., "PPAR gamma 2 prevents lipotoxicity by controlling adipose tissue expandability and peripheral lipid metabolism," PLoS Genetics, vol. 3, no. 4, article e64, pp. 634-647, 2007.

[34] A. E. Caballero, R. Saouaf, S. C. Lim et al., "The effects of troglitazone, an insulin-sensitizing agent, on the endothelial function in early and late type 2 diabetes: a placebo-controlled randomized clinical trial," Metabolism: Clinical and Experimental, vol. 52, no. 2, pp. 173-180, 2003.

[35] T. M. Larsen, S. Toubro, and A. Astrup, "PPARgamma agonists in the treatment of type II diabetes: is increased fatness commensurate with long-term efficacy?" International Journal of Obesity, vol. 27, no. 2, pp. 147-161, 2003.

[36] N. Marx, T. Bourcier, G. K. Sukhova, P. Libby, and J. Plutzky, "PPAR $\gamma$ activation in human endothelial cells increases plasminogen activator inhibitor type- 1 expression: $\operatorname{PPAR} \gamma$ as a potential mediator in vascular disease," Arteriosclerosis, Thrombosis, and Vascular Biology, vol. 19, no. 3, pp. 546-551, 1999.

[37] A. Raji, E. W. Seely, S. A. Bekins, G. H. Williams, and D. C. Simonson, "Rosiglitazone improves insulin sensitivity and lowers blood pressure in hypertensive patients," Diabetes Care, vol. 26, no. 1, pp. 172-178, 2003.

[38] J. Ablain and L. I. Zon, "Of fish and men: using zebrafish to fight human diseases," Trends in Cell Biology, vol. 23, no. 12, pp. 584586, 2013.

[39] T. Cermak, E. L. Doyle, M. Christian et al., "Efficient design and assembly of custom TALEN and other TAL effector-based constructs for DNA targeting," Nucleic Acids Research, vol. 39, no. 12, article e82, 2011.

[40] W. Y. Hwang, Y. Fu, D. Reyon et al., "Efficient genome editing in zebrafish using a CRISPR-Cas system," Nature Biotechnology, vol. 31, no. 3, pp. 227-229, 2013.

[41] G. N. Wheeler and A. W. Brändli, "Simple vertebrate models for chemical genetics and drug discovery screens: lessons from zebrafish and Xenopus," Developmental Dynamics, vol. 238, no. 6, pp. 1287-1308, 2009.

[42] M. Tsang, "Zebrafish: a tool for chemical screens," Birth Defects Research Part C-Embryo Today: Reviews, vol. 90, no. 3, pp. 185192, 2010.

[43] R. T. Peterson and M. C. Fishman, "Designing Zebrafish chemical screens," in Methods in Cell Biology, vol. 105, chapter 23, pp. 525-541, Elsevier, 2011.

[44] A. Schlegel and D. Y. R. Stainier, "Lessons from 'lower' organisms: what worms, flies, and zebrafish can teach us about human energy metabolism," PLoS Genetics, vol. 3, no. 11, article e199, 2007.

[45] T. Oka, Y. Nishimura, L. Zang et al., "Diet-induced obesity in zebrafish shares common pathophysiological pathways with mammalian obesity," BMC Physiology, vol. 10, article 21, 2010.

[46] J. L. Anderson, J. D. Carten, and S. A. Farber, "Zebrafish lipid metabolism: from mediating early patterning to the metabolism of dietary fat and cholesterol," Methods in Cell Biology, vol. 101, pp. 111-141, 2011.

[47] S.-I. Nishio, Y. Gibert, L. Berekelya et al., "Fasting induces CART down-regulation in the zebrafish nervous system in a cannabinoid receptor 1-dependent manner," Molecular Endocrinology, vol. 26, no. 8, pp. 1316-1326, 2012.

[48] V. Jiménez-Amilburu, S. Jong-Raadsen, J. Bakkers, H. P. Spaink, and R. Marín-Juez, "GLUT12 deficiency during early development results in heart failure and a diabetic phenotype in zebrafish," Journal of Endocrinology, vol. 224, no. 1, pp. 1-15, 2015.

[49] M. R. Dalman, Q. Liu, M. D. King, B. Bagatto, and R. L. Londraville, "Leptin expression affects metabolic rate in zebrafish embryos (D. Rerio)," Frontiers in Physiology, vol. 4, article 160, 2013.

[50] E. J. Flynn, C. M. Trent, and J. F. Rawls, "Ontogeny and nutritional control of adipogenesis in zebrafish (Danio rerio)," Journal of Lipid Research, vol. 50, no. 8, pp. 1641-1652, 2009. 
[51] D. Imrie and K. C. Sadler, "White adipose tissue development in zebrafish is regulated by both developmental time and fish size," Developmental Dynamics, vol. 239, no. 11, pp. 3013-3023, 2010.

[52] A. G. Little and F. Seebacher, "The evolution of endothermy is explained by thyroid hormone-mediated responses to cold in early vertebrates," Journal of Experimental Biology, vol. 217, no. 10, pp. 1642-1648, 2014.

[53] Y.-C. Tseng, R.-D. Chen, M. Lucassen et al., "Exploring uncoupling proteins and antioxidant mechanisms under acute cold exposure in brains of fish," PLOS ONE, vol. 6, no. 3, Article ID e18180, 2011.

[54] M. Hölttä-Vuori, V. T. V. Salo, L. Nyberg et al., "Zebrafish: gaining popularity in lipid research," Biochemical Journal, vol. 429 , no. 2, pp. 235-242, 2010.

[55] J. H. Lee, J.-H. So, J. H. Jeon et al., "Synthesis of a new fluorescent small molecule probe and its use for in vivo lipid imaging," Chemical Communications, vol. 47, no. 26, pp. 7500-7502, 2011.

[56] H. S. Chun, J. H. Jeon, H. S. Pagire et al., "Synthesis of LipidGreen2 and its application in lipid and fatty liver imaging," Molecular Biosystems, vol. 9, no. 4, pp. 630-633, 2013.

[57] S. Fukumoto and T. Fujimoto, "Deformation of lipid droplets in fixed samples," Histochemistry and Cell Biology, vol. 118, no. 5, pp. $423-428,2002$.

[58] A. Tingaud-Sequeira, N. Ouadah, and P. J. Babin, "Zebrafish obesogenic test: a tool for screening molecules that target adiposity," Journal of Lipid Research, vol. 52, no. 9, pp. 1765-1772, 2011.

[59] K. W. Park, D. S. Halperin, and P. Tontonoz, "Before they were fat: adipocyte progenitors," Cell Metabolism, vol. 8, no. 6, pp. 454-457, 2008.

[60] Q. Q. Tang and M. D. Lane, "Adipogenesis: from stem cell to adipocyte," Annual Review of Biochemistry, vol. 81, no. 1, pp. 715736, 2012.

[61] S. R. Farmer, "Transcriptional control of adipocyte formation," Cell Metabolism, vol. 4, no. 4, pp. 263-273, 2006.

[62] D. P. Ramji and P. Foka, "CCAAT/enhancer-binding proteins: structure, function and regulation," Biochemical Journal, vol. 365, no. 3, pp. 561-575, 2002.

[63] S.-I. Nishio, Y. Gibert, L. Bernard, F. Brunet, G. Triqueneaux, and V. Laudet, "Adiponectin and adiponectin receptor genes are coexpressed during zebrafish embryogenesis and regulated by food deprivation," Developmental Dynamics, vol. 237, no. 6, pp. 1682-1690, 2008.

[64] T. Yanagiya, A. Tanabe, and K. Hotta, "Gap-junctional communication is required for mitotic clonal expansion during adipogenesis," Obesity, vol. 15, no. 3, pp. 572-582, 2007.

[65] A. Tingaud-Sequeira, A. Knoll-Gellida, M. André, and P. J. Babin, "Vitellogenin expression in white adipose tissue in female teleost fish," Biology of Reproduction, vol. 86, no. 2, article 38, 2012.

[66] H. Keller, C. Dreyer, J. Medin, A. Mahfoudi, K. Ozato, and W. Wahli, "Fatty acids and retinoids control lipid metabolism through activation of peroxisome proliferatoractivated receptor-retinoid X receptor heterodimers," Proceedings of the National Academy of Sciences of the United States of America, vol. 90, no. 6, pp. 2160-2164, 1993.

[67] S. A. Kliewer, S. S. Sundseth, S. A. Jones et al., "Fatty acids and eicosanoids regulate gene expression through direct interactions with peroxisome proliferator-activated receptors $\alpha$ and $\gamma$," Proceedings of the National Academy of Sciences of the United States of America, vol. 94, no. 9, pp. 4318-4323, 1997.
[68] P. R. Devchand, H. Keller, J. M. Peters, M. Vazquez, F. J. Gonzalez, and W. Wahli, "The PPAR $\alpha$-leukotriene B4 pathway to inflammation control," Nature, vol. 384, no. 6604, pp. 39-43, 1996.

[69] K. Yu, W. Bayona, C. B. Kallen et al., "Differential activation of peroxisome proliferator-activated receptors by eicosanoids," Journal of Biological Chemistry, vol. 270, no. 41, pp. 2397523983, 1995.

[70] S. A. Kliewer, J. M. Lenhard, T. M. Willson, I. Patel, D. C. Morris, and J. M. Lehmann, "A prostaglandin J2 metabolite binds peroxisome proliferator-activated receptor gamma and promotes adipocyte differentiation," Cell, vol. 83, no. 5, pp. 813819, 1995.

[71] S. E. O’Sullivan, "Cannabinoids go nuclear: evidence for activation of peroxisome proliferator-activated receptors," British Journal of Pharmacology, vol. 152, no. 5, pp. 576-582, 2007.

[72] J. M. Lehmann, J. M. Lenhard, B. B. Oliver, G. M. Ringold, and S. A. Kliewer, "Peroxisome proliferator-activated receptors $\alpha$ and $\gamma$ are activated by indomethacin and other non-steroidal antiinflammatory drugs," The Journal of Biological Chemistry, vol. 272, no. 6, pp. 3406-3410, 1997.

[73] S. Kersten, B. Desvergne, and W. Wahli, "Roles of PPARS in health and disease," Nature, vol. 405, no. 6785, pp. 421-424, 2000.

[74] T. M. Willson, P. J. Brown, D. D. Sternbach, and B. R. Henke, "The PPARs: from orphan receptors to drug discovery," Journal of Medicinal Chemistry, vol. 43, no. 4, pp. 527-550, 2000.

[75] A. Chawla, C.-H. Lee, Y. Barak et al., "PPARdelta is a very lowdensity lipoprotein sensor in macrophages," Proceedings of the National Academy of Sciences of the United States of America, vol. 100, no. 3, pp. 1268-1273, 2003.

[76] R. A. Ngala, C. J. Stocker, A. G. Roy et al., "A new, highly selective murine peroxisome proliferator-activated receptor $\delta$ agonist increases responsiveness to thermogenic stimuli and glucose uptake in skeletal muscle in obese mice," Diabetes, Obesity and Metabolism, vol. 13, no. 5, pp. 455-464, 2011.

[77] W. R. Oliver Jr., J. L. Shenk, M. R. Snaith et al., "A selective peroxisome proliferator-activated receptor $\delta$ agonist promotes reverse cholesterol transport," Proceedings of the National Academy of Sciences of the United States of America, vol. 98, no. 9, pp. 5306-5311, 2001.

[78] B. M. Forman, P. Tontonoz, J. Chen, R. P. Brun, B. M. Spiegelman, and R. M. Evans, "15-deoxy- $\Delta^{12}$, ${ }^{14}$-prostaglandin $J_{2}$ is a ligand for the adipocyte determination factor PPAR $\gamma$," Cell, vol. 83, no. 5, pp. 803-812, 1995.

[79] J. M. Lehmann, L. B. Moore, T. A. Smith-Oliver, W. O. Wilkison, T. M. Willson, and S. A. Kliewer, "An antidiabetic thiazolidinedione is a high affinity ligand for peroxisome proliferatoractivated receptor $\gamma$ (PPAR $\gamma$ )," The Journal of Biological Chemistry, vol. 270, no. 22, pp. 12953-12956, 1995.

[80] S. Bertrand, B. Thisse, R. Tavares et al., "Unexpected novel relational links uncovered by extensive developmental profiling of nuclear receptor expression," PLoS Genetics, vol. 3, no. 11, article e188, 2007.

[81] J. M. McPartland, M. Glass, I. Matias, R. W. Norris, and C. W. Kilpatrick, "A shifted repertoire of endocannabinoid genes in the zebrafish (Danio rerio)," Molecular Genetics and Genomics, vol. 277, no. 5, pp. 555-570, 2007.

[82] Y. Zhao, K. Zhang, J. P. Giesy, and J. Hu, "Families of nuclear receptors in vertebrate models: characteristic and comparative toxicological perspective," Scientific Reports, vol. 5, article 8554, 2015. 
[83] M. A. Gates, L. Kim, E. S. Egan et al., "A genetic linkage map for zebrafish: comparative analysis and localization of genes and expressed sequences," Genome Research, vol. 9, no. 4, pp. 334347, 1999.

[84] O. Jaillon, J.-M. Aury, F. Brunet et al., "Genome duplication in the teleost fish Tetraodon nigroviridis reveals the early vertebrate proto-karyotype," Nature, vol. 431, no. 7011, pp. 946957, 2004.

[85] J. M. Catchen, J. S. Conery, and J. H. Postlethwait, "Automated identification of conserved synteny after whole-genome duplication," Genome Research, vol. 19, no. 8, pp. 1497-1505, 2009.

[86] L. Michalik, B. Desvergne, C. Dreyer, M. Gavillet, R. N. Laurini, and W. Wahli, "PPAR expression and function during vertebrate development," International Journal of Developmental Biology, vol. 46, no. 1, pp. 105-114, 2002.

[87] T. Zhou, X. Yan, G. Wang et al., "Evolutionary pattern and regulation analysis to support why diversity functions existed within PPAR gene family members," BioMed Research International, vol. 2015, Article ID 613910, 11 pages, 2015.

[88] H. Escriva, F. Delaunay, and V. Laudet, "Ligand binding and nuclear receptor evolution," BioEssays, vol. 22, no. 8, pp. 717727, 2000.

[89] A. Riu, M. Grimaldi, A. le Maire et al., "Peroxisome proliferatoractivated receptor $\gamma$ is a target for halogenated analogs of bisphenol A," Environmental Health Perspectives, vol. 119, no. 9, pp. 1227-1232, 2011. 


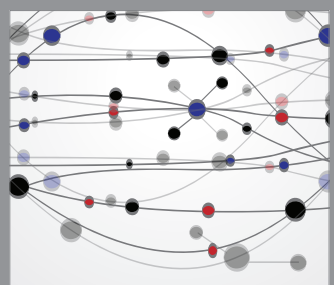

The Scientific World Journal
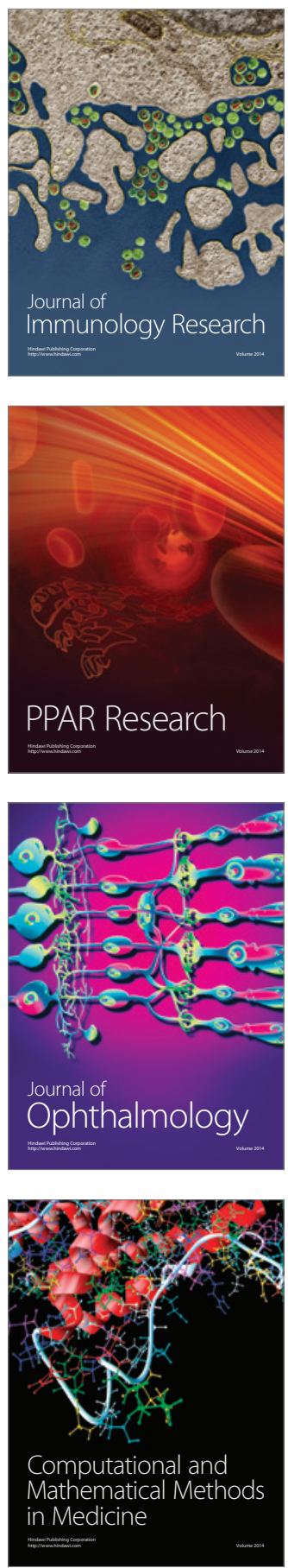

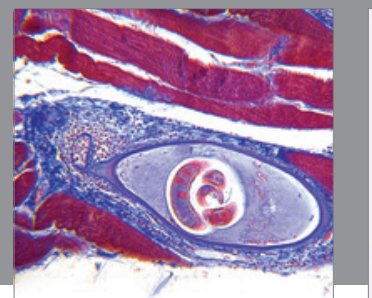

Gastroenterology

Research and Practice
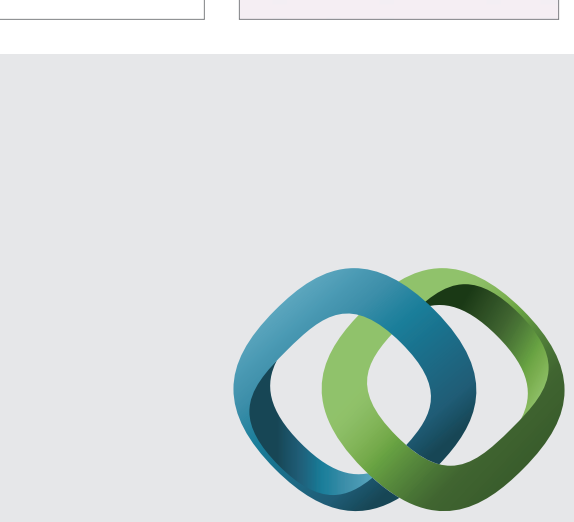

\section{Hindawi}

Submit your manuscripts at

http://www.hindawi.com
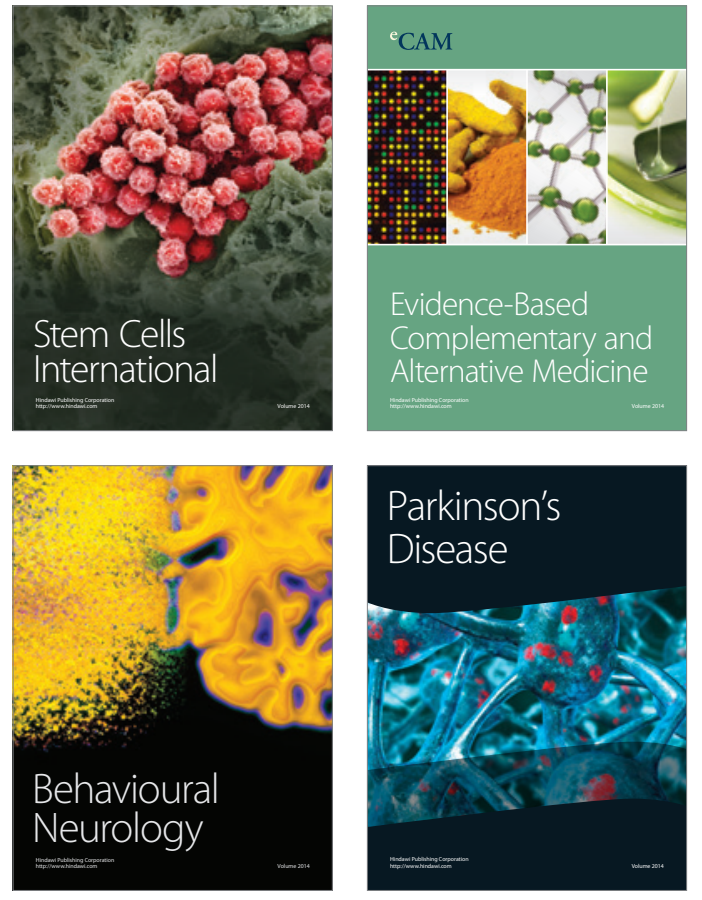
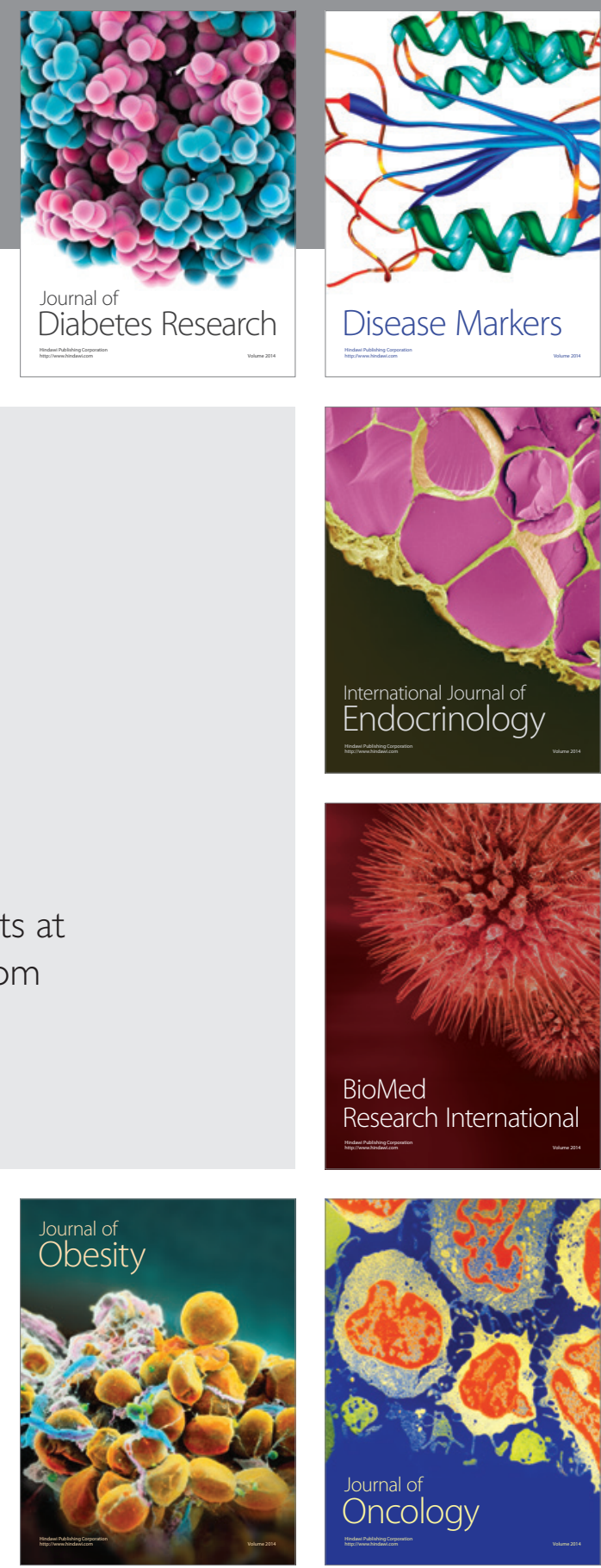

Disease Markers
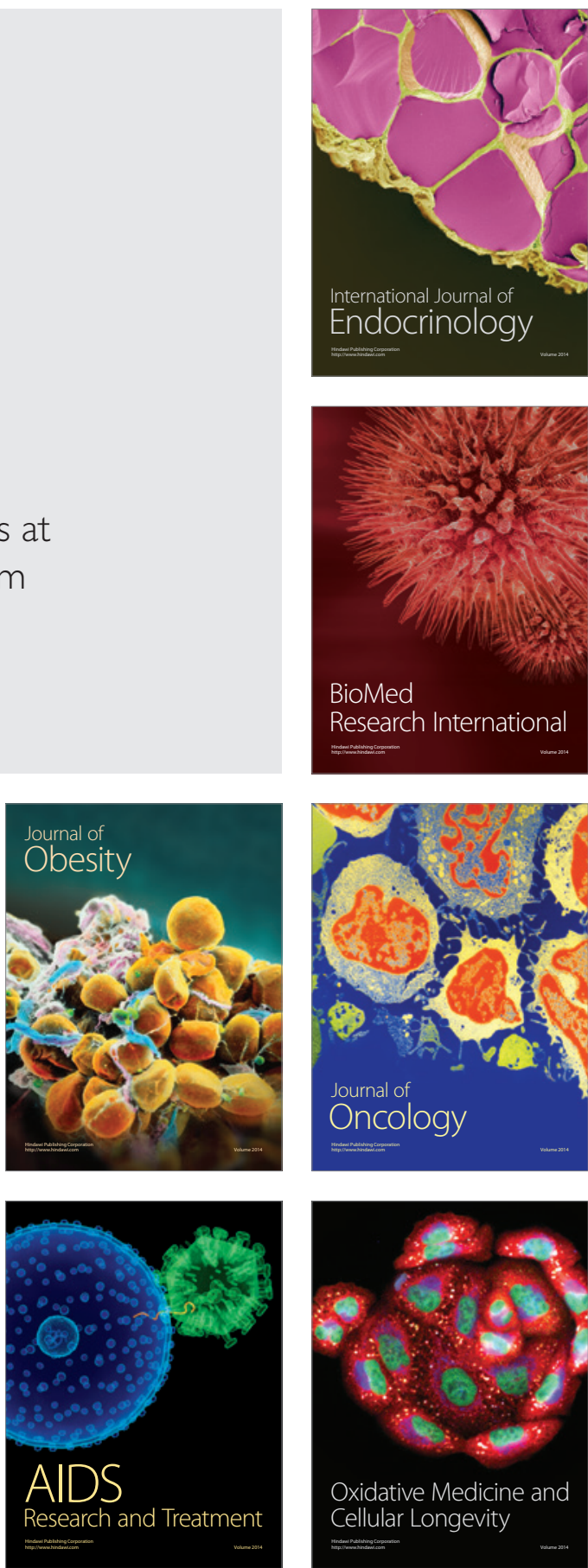